\title{
Temperature effects on quantum interference in molecular junctions
}

\author{
Markussen, Troels; Thygesen, Kristian Sommer
}

Published in:

Physical Review B

Link to article, DOI:

10.1103/PhysRevB.89.085420

Publication date:

2014

Document Version

Publisher's PDF, also known as Version of record

Link back to DTU Orbit

Citation (APA):

Markussen, T., \& Thygesen, K. S. (2014). Temperature effects on quantum interference in molecular junctions. Physical Review B, 89(8), 085420. https://doi.org/10.1103/PhysRevB.89.085420

\section{General rights}

Copyright and moral rights for the publications made accessible in the public portal are retained by the authors and/or other copyright owners and it is a condition of accessing publications that users recognise and abide by the legal requirements associated with these rights.

- Users may download and print one copy of any publication from the public portal for the purpose of private study or research.

- You may not further distribute the material or use it for any profit-making activity or commercial gain

- You may freely distribute the URL identifying the publication in the public portal

If you believe that this document breaches copyright please contact us providing details, and we will remove access to the work immediately and investigate your claim. 


\title{
Temperature effects on quantum interference in molecular junctions
}

\author{
Troels Markussen* and Kristian S. Thygesen \\ Center for Atomic-scale Materials Design (CAMD), Department of Physics, Technical University of Denmark, \\ DK-2800 Kongens Lyngby, Denmark \\ (Received 9 September 2013; revised manuscript received 9 February 2014; published 21 February 2014)
}

\begin{abstract}
A number of experiments have demonstrated that destructive quantum interference (QI) effects in molecular junctions lead to very low conductances even at room temperature. On the other hand, another recent experiment showed increasing conductance with temperature which was attributed to decoherence effects destroying QI at finite temperatures. Here we study the influence of finite temperatures and electron-phonon interactions on QI in molecular junctions. Two different models leading to two inherently different types of QI effects are considered. Each model is exemplified by specific molecules and studied using first-principles calculations. We find that the molecules exhibiting QI show a much stronger temperature dependence of the conductance compared to molecules without QI. However, the large QI-induced suppression of the conductance remains, showing that QI effects are indeed robust against finite temperatures and inelastic scattering.
\end{abstract}

DOI: 10.1103/PhysRevB.89.085420

PACS number(s): 85.65.+h, 72.10.Di, 73.23.-b

\section{INTRODUCTION}

A number of recent experiments have demonstrated the importance of destructive quantum interference (QI) effects in different molecular junctions leading to very low conductances when compared to analogous junctions without QI effects [1-6]. These experiments, which are all conducted at room temperature at relatively low-bias voltages, confirm earlier experimental works on both electron transport and transfer in benzene-based molecules [7,8]. QI effects demonstrate some intriguing possibilities in molecular electronics, where small structural or chemical changes can lead to pronouncedly different transport properties. Molecules exhibiting destructive QI were recently used as the basis for ultrathin insulating molecular films [6], and for electrochemically controlled molecular switches $[10,11]$. Other applications within thermoelectrics have recently been proposed [12-15].

Common to the experimentally studied molecules $[1-6,8,9]$ showing QI effects is that they are cross conjugated with up to several orders of magnitude lower conductance than linear conjugated analogous molecules. A molecule in connection with two leads is termed linearly conjugated if one can draw a path connecting the two leads, which strictly alternate between single and double or triple bonds. A pathway is cross conjugated if it contains two subsequent single bonds and the $\left(s p^{2}\right.$ hybridized) carbon atom linking these single bonds is double bonded to any group or atom in a third direction [16]. A molecule is called cross conjugated if all the pathways are cross conjugated. A large number of theoretical works have predicted QI effects to be present in cross-conjugated molecules but not in linearly conjugated ones [10,17-19]. In simplified models considering only the $\pi$ system, the cross-conjugated molecules will exhibit a transmission node at an energy close to the Fermi energy, leading to a very low conductance [19]. For more details on the relation between QI and conjugation patterns, we refer to Ref. [9].

\footnotetext{
*Present address: QuantumWise A/S, Lersø Parkalle 107, Copenhagen $\emptyset$, DK-2100, Denmark.
}

Recent experiments [20], conducted at large-bias voltages in a resonant transport situation, showed a significant temperature dependence of the conductance of the molecule 2,2'-dimethylbiphenyl (DMBP). The observed increase in conductance with temperature was attributed to decoherence processes destroying a QI effect due to quasidegenerate molecular energy levels. Previous theoretical works [21,22] showed indeed that at high-bias voltages electron-phonon interactions lead to strong effects on systems with quasidegenerate levels. This is interesting since typically electron-phonon interactions only lead to a modest effect on the electronic conductance in molecular junctions [23] with a conductance change of a few percent as measured by, e.g., inelastic tunneling spectroscopy (IETS) and in agreement with first-principles calculations [24].

The conclusions drawn from Refs. [20-22] indicate that QI effects could be destroyed at high (room) temperatures. At first sight this may seem inconsistent with the experimental findings in Refs. [1-6], and calls for further theoretical studies.

In this paper we consider finite temperature effects on the conductance in a molecular junction showing QI effects. We consider both the simple effect of temperature broadening of the Fermi distribution functions and inelastic scattering effects due to electron-phonon (el-ph) interactions. We first identify the two simplest topologically different models leading to QI effects, and we exemplify each model with a real molecule. For studying el-ph effects we use density functional theory (DFT) and diagrammatic perturbation theory. We find that the el-ph interactions lead to strong temperature-dependent conductances for systems with QI effects. However, QI effects are not at all completely destroyed and at room temperature we still observe an orders of magnitude difference in conductance between cross-conjugated molecules and linearly conjugated molecules, in agreement with experimental findings.

\section{METHODS}

We consider a molecule $(M)$ coupled to a left $(L)$ and right $(R)$ electrode described with an electronic Hamiltonian of the form

$$
\mathbf{H}=\mathbf{H}_{L}+\mathbf{V}_{L M}+\mathbf{H}_{M}+\mathbf{V}_{R M}+\mathbf{H}_{R},
$$


where $\mathbf{H}_{L}\left(\mathbf{H}_{R}\right)$ describes noninteracting electrons in the left (right) electrodes, and $\mathbf{V}_{\alpha M}$ is the coupling between the molecule and the electrodes. In this paper we focus on intrinsic properties of the molecules and not on the details in the molecule-electrode coupling. Hence, we describe the electrodes within a wide-band approximation, giving rise to constant, purely imaginary electrode self-energies. The molecular Hamiltonian $\mathbf{H}_{M}$ depends on the nuclear coordinates $\bar{R}=\bar{R}_{0}+\bar{Q}$, where $\bar{R}_{0}$ is the equilibrium, zero-temperature configuration, and $\bar{Q}$ is a displacement vector. For small displacements we may write

$$
\mathbf{H}_{M}=\mathbf{H}_{M}^{0}+\sum_{\lambda} \mathbf{M}_{\lambda}\left(b_{\lambda}^{\dagger}+b_{\lambda}\right),
$$

where $\mathbf{H}_{M}^{0}$ is the Hamiltonian corresponding to the ionic coordinates $\bar{R}=\bar{R}_{0} \cdot \mathbf{H}_{M}^{0}$ is taken as the Kohn-Sham Hamiltonian evaluated in a localized atomic orbital basis as implemented in the GPAW code [25]. $b_{\lambda}^{\dagger}$ and $b_{\lambda}$ are the creation and annihilation operators of phonon mode $\lambda$, and $\mathbf{M}_{\lambda}$ is the el-ph coupling matrix. We calculate the phonon modes as well as the el-ph coupling matrices for free molecules using finite difference methods as implemented in GPAW [26].

\section{A. Self-consistent Born approximation (SCBA)}

In order to address the effect of el-ph interactions we use the nonequilibrium Green's function (NEGF) formalism. The el-ph interactions are treated perturbatively within the self-consistent Born approximation (SCBA) [23,24]. In the solution to the SCBA equations we use the free phonon Green's functions and further assume that the phonon mode occupations are given by the Bose-Einstein distribution, thus corresponding to an externally damped situation [24]. We only include the Fock diagram in the electron-phonon selfenergy, as the Hartree self-energy merely leads to a biasand temperature-independent energy shift. The current from the left lead through the molecules is calculated using the Meir-Wingreen formula [27]

$$
I_{L}(V)=\frac{2 e}{h} \int_{-\infty}^{\infty} d \varepsilon \operatorname{Tr}\left[\boldsymbol{\Sigma}_{L}^{<}(\varepsilon) \mathbf{G}^{>}(\varepsilon)-\boldsymbol{\Sigma}_{L}^{>}(\varepsilon) \mathbf{G}^{<}(\varepsilon)\right],
$$

where $\mathbf{G}^{<}\left(\mathbf{G}^{>}\right)$is the electronic lesser (greater) Green's function for the molecule, and $\boldsymbol{\Sigma}_{L}^{<}\left(\boldsymbol{\Sigma}_{L}^{>}\right)$is the lesser (greater) self-energy due to coupling to the left electrode. A similar expression for the right-going current is obtained by replacing $\Sigma_{L}^{<,>}$with $\Sigma_{R}^{<,>}$. Since SCBA is a current conserving approximation we have $I_{L}=-I_{R}=I$. In addition to the total current given by Eq. (3), we also calculate a purely elastic current as

$$
I_{e l}(V)=\frac{2 e}{h} \int_{-\infty}^{\infty} \mathcal{T}(\varepsilon)\left[f\left(\mu_{L}, T\right)-f\left(\mu_{R}, T\right)\right] d \varepsilon,
$$

where $f(\mu, T)$ is the Fermi-Dirac distribution function at chemical potential $\mu$ and temperature $T$, and

$$
\mathcal{T}(\varepsilon)=\operatorname{Tr}\left[\mathbf{G}^{r} \boldsymbol{\Gamma}_{\mathbf{L}} \mathbf{G}^{a} \boldsymbol{\Gamma}_{\mathbf{R}}\right](\varepsilon)
$$

is the transmission function calculated from the interacting retarded and advanced Green's functions and $\Gamma_{L, R}=$ $-2 \operatorname{Im} \Sigma_{L, R}^{r}=0.2 \mathrm{eV}$. We define the inelastic contribution to the current as $I_{\mathrm{in}}=I-I_{\mathrm{el}}$. If in Eqs. (4) and (5) we use the noninteracting Green's functions, we obtain the noninteracting current $I_{0}$. We have found that essentially $I_{0} \approx I_{\mathrm{el}}$ and below we only show results for $I_{0}$.

\section{B. Lowest order expansion (LOE)}

In addition to the full solution to SCBA, we also apply a lowest order expansion (LOE) of Eq. (3) [24,28], which avoids self-consistency loops and where the Green's functions are evaluated only around the Fermi energy. The LOE approximation relies on the assumption that the electronic Green's functions vary slowly around the Fermi energy on the energy scale of the phonon energy $\hbar \omega$. The current for a symmetric junction can be expressed as

$$
\begin{aligned}
I^{\mathrm{LOE}}(V)= & I_{0}(V) \\
& +\sum_{\lambda} I_{\lambda}^{\mathrm{sym}}\left(V, T,\left\langle n_{\lambda}\right\rangle\right) \\
& \times \operatorname{Tr}\left[\mathbf { G } _ { 0 } ^ { a } \boldsymbol { \Gamma } _ { L } \mathbf { G } _ { 0 } ^ { r } \left\{\mathbf{M}^{\lambda} \mathbf{A}_{0, R} \mathbf{M}^{\lambda}\right.\right. \\
& \left.\left.+\frac{i}{2}\left(\boldsymbol{\Gamma}_{R} \mathbf{G}_{0}^{a} \mathbf{M}^{\lambda} \mathbf{A}_{0} \mathbf{M}^{\lambda}-\text { H.c. }\right)\right\}\right],
\end{aligned}
$$

where

$$
I_{\lambda}^{\mathrm{sym}}=\frac{e}{\pi \hbar}\left(2 e V\left\langle n_{\lambda}\right\rangle+\frac{\hbar \omega_{\lambda}-e V}{e^{\beta\left(\hbar \omega_{\lambda}-e V\right)}-1}-\frac{\hbar \omega_{\lambda}+e V}{e^{\beta\left(\hbar \omega_{\lambda}+e V\right)}-1}\right) .
$$

Here $\mathbf{G}_{0}$ refers to the Green's function in the absence of electron-phonon coupling. Further, $\mathbf{A}_{0}=i\left(\mathbf{G}_{0}^{r}-\mathbf{G}_{0}^{a}\right)$ and $\mathbf{A}_{0, R}=\mathbf{G}_{0}^{r} \boldsymbol{\Gamma}_{R} \mathbf{G}_{0}^{a}$. The LOE is a current conserving approximation and, as shown in Appendix A, the LOE provides a very good approximation to the SCBA results for the models we consider here, in agreement with previous work on molecular transport in the low-bias regime [24,28].

\section{RESULTS}

\section{A. Two models with QI effects}

The experimentally studied molecules showing QI effects can be divided into two classes: In the first class the transmission function for the $\pi$ electrons has a node close to the Fermi energy, due to destructive interference effects between the highest occupied molecular orbitals (HOMOs) and the lowest unoccupied molecular orbitals (LUMOs) [1-6]. These molecules are all cross conjugated. A simple generic example of a molecular junction falling in this class is metaconnected benzene, shown in Fig. 1(a). The metaconnected benzene is attractive due to its simple structure, but is difficult to realize in practice. A different, experimentally more relevant molecule belonging to this class is anthraquinone (AQ). In this paper we take meta-connected benzene and AQ as paradigmatic examples of the molecules in the first QI class. For comparison we study the linearly conjugated anthracene (AC). The AC does not present $\mathrm{QI}$ and is obtained from AQ by replacing the two oxygen atoms on the central ring by hydrogen.

In the second class of molecules, the QI effect occur due to two quasidegenerate levels, e.g., HOMO and HOMO-1 [21]. A molecular realization of this group is $2,2^{\prime}$-dimethylbiphenyl 
(a)
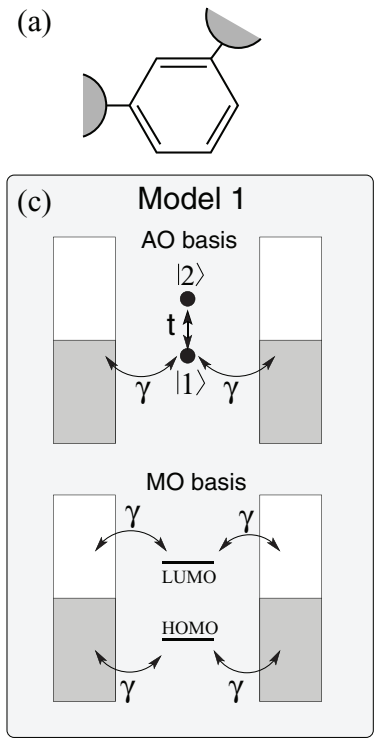

(e)

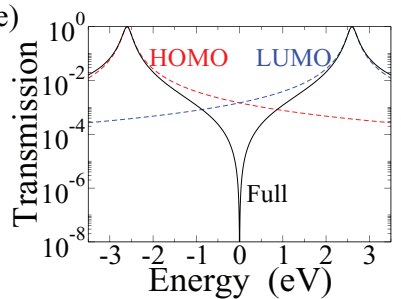

(b)
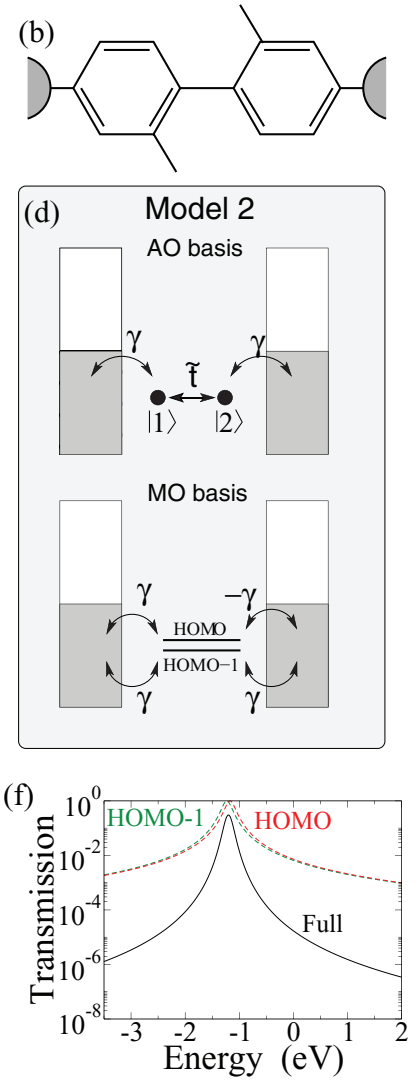

FIG. 1. (Color online) Two models showing different types of QI, each represented by a concrete molecule: (a) metabenzene and (b) DMBP (b). (c) and (d) show schematically the two models in an "atomic orbital" (AO) representation illustrating a real-space distribution of the two sites, and in a molecular orbital (MO) representation showing the MO energy levels. The AO on-site energies are set to $\varepsilon_{1}=0$ for model 1 and $\varepsilon_{2}=-1.2 \mathrm{eV}$ for model 2. (e) and (f) show transmission functions for the two models (black lines), together with transmission functions from independent (i.e., without QI) MO levels.

(DMBP), shown in Fig. 1(b). This molecule (apart from additional spacer groups) was experimentally studied in Ref. [20], showing evidence of QI as witnessed by a strong temperature-dependent current. In this paper we use DMBP as a concrete example of a molecule exhibiting QI of the second type.

In Figs. 1(c) and 1(d) we show the simplest topological models presenting QI of the two generic types described above. Both models are illustrated using an atomic orbital (AO) representation illustrating a real-space distribution of the two sites $|1\rangle$ and $|2\rangle$ and using a molecular orbital (MO) representation showing the MO energy levels. In model 1, the two MOs could for example be the HOMO and LUMO of benzene. In the AO basis, the two sites $|1\rangle$ and $|2\rangle$ are coupled with a large hopping amplitude, $t=-2.6 \mathrm{eV}$, but only site $|1\rangle$ is coupled to the electrodes through coupling parameter $\gamma$ (see also Appendix B). Figure 1(e) shows the noninteracting transmission function obtained with model 1 . The transmission node at energy $E=0 \mathrm{eV}$ is a characteristic feature of QI in this kind of model. Figure 1(e) also shows transmission functions

obtained from the independent MO levels (dashed lines), i.e., a single site model with either only the HOMO or only the LUMO. We define here QI as the difference in transmission between the solid line including both levels and the sum of the two dashed lines. Right at the transmission node at $E=0 \mathrm{eV}$ there is completely destructive QI.

In model 2 we consider only the DMBP HOMO and HOMO-1 levels, which are separated in energy by a small $\Delta \varepsilon$. The two benzene rings in DMBP are almost orthogonal (with a tilt angle of $81^{\circ}$ ), leading to a weak coupling between the $\pi$ systems on the two benzene rings. In the AO basis this leads to left and right localized states with a weak coupling $\tilde{t}=-\Delta \varepsilon / 2=-0.03 \mathrm{eV}$. Due to the weak coupling, the transmission function shown in Fig. 1(f) is clearly suppressed when compared to the independent HOMO and HOMO-1 levels. Model 2 exhibits no completely destructive QI as model 1 , except in the trivial limit of $\tilde{t}=0$.

We now consider the effect of el-ph interactions on models 1 and 2. From the DFT calculated phonon modes and electron-phonon couplings we have found that a single phonon mode is clearly dominating the scattering for both benzene and DMPB. The relevant phonon energies are $\hbar \omega_{1}=0.073$ $\mathrm{meV}$ for benzene and $\hbar \omega_{2}=0.005 \mathrm{eV}$ for DMPB. The latter corresponds to a torsional mode around the central bond. From DFT calculations we obtain the el-ph coupling matrices written in the $\mathrm{AO}$ basis as

$$
\mathbf{M}^{(1)}=\left(\begin{array}{ll}
m_{1} & m_{1} \\
m_{1} & m_{1}
\end{array}\right), \quad \mathbf{M}^{(2)}=\left(\begin{array}{cc}
0 & m_{2} \\
m_{2} & 0
\end{array}\right),
$$

with $m_{1}=0.060 \mathrm{eV}$ and $m_{2}=0.047 \mathrm{eV}$. We note that for DMBP we obtain a very strong electron-phonon interaction strength with $m_{2} / \hbar \omega_{2}=9.4$. The use of diagrammatic perturbation theory is strictly valid only for $m / \hbar \omega<1$. While this may affect the quantitative results we obtain for DMBP and model 2, we note that the current obtained after one iteration of the SCBA equations typically differs by less than $10 \%$ of the self-consistent current. We thus expect the main conclusions to remain valid. An alternative approach based on a small polaron transformation was taken in Refs. [21,22]. For benzene and model $1, m_{1} / \hbar \omega_{1}=0.8$, and perturbation theory is applicable.

When el-ph interactions are included, the electronic energy levels are renormalized by a so-called polaron shift $\Delta E=$ $|\langle\psi|M| \psi\rangle|^{2} / \hbar \omega$. In the SCBA this constant shift is included in the Hartree diagram, which we do not include in our SCBA calculations nor in the LOE results. While inclusion of the Hartree diagram leads to a modified current at a given bias, the differential conductance $d I / d V$ and the relative conductance change with temperatures are essentially unaffected.

\section{B. Temperature-dependent current}

Figure 2 shows current versus temperature calculated for model 1 (left column) and model 2 (right column) at bias voltages $V_{B}=0.01 \mathrm{~V}$ (top) and $V_{B}=0.5 \mathrm{~V}$ (bottom). At low-bias voltage we observe a strong temperature dependence for model 1. At low temperatures the phonon mode is not thermally excited and the inelastic current (dotted blue) is zero. For temperatures above $100 \mathrm{~K}$, the phonon mode becomes thermally excited and the inelastic current increases. At the high-bias voltage, however, we see that model 1 is dominated 


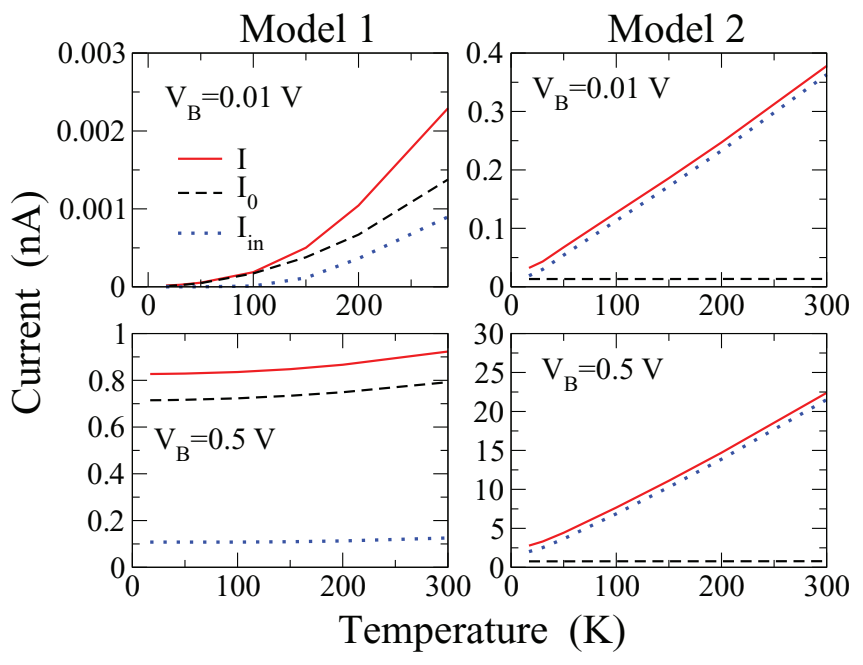

FIG. 2. (Color online) Current vs temperature for model 1 (left column) and model 2 (right column). The applied bias voltage is $V_{B}=0.01 \mathrm{~V}$ (top row) and $V_{B}=0.5 \mathrm{~V}$ (bottom row). In each plot we show the noninteracting current (dashed black), the total current including el-ph interactions (solid red), and the inelastic contribution (dotted blue).

by the elastic (noninteracting) contribution to the current (dashed black), and the effects of el-ph interactions are rather small.

The situation is completely different for model 2 , where the current is almost completely dominated by the inelastic contribution, even at low temperatures. The low phonon energy in model 2 implies that both phonon emission and absorption processes contribute to the current even at low temperatures and small-bias voltages. When the torsional mode is excited, the $\pi$ systems on the two benzene rings will on average be less orthogonal, and the effective coupling between them will be larger, resulting in a larger current. The approximately linearly increasing current versus temperature can be derived analytically from the LOE approximation. Within the LOE, the temperature dependence is determined by (i) the temperature dependence of the noninteracting conductance and (ii) the variation of $I^{\text {sym }}$ given by Eq. (7). Applying a bias such that $e V \gg \hbar \omega_{\lambda}$ and $e V \gg k_{B} T$, the last term in Eq. (7) vanishes and the middle term approximately becomes $e V$, and is independent of temperature. Assuming that the occupation $\left\langle n_{\lambda}\right\rangle$ of the phonon mode $\lambda$ is given by the Bose-Einstein distribution $\left\langle n_{\lambda}\right\rangle=1 /\left(e^{\hbar \omega_{\lambda} / k_{B} T}-1\right)$, we get at temperatures $k_{B} T>\hbar \omega$ that $\left\langle n_{\lambda}\right\rangle \approx \frac{k_{B} T}{\hbar \omega}$, and the LOE contribution to the conductance is thus seen to approximately increase linearly with temperature, in accordance with the full solution to SCBA for model 2 in Fig. 2.

We note that the strong temperature dependence and importance of the inelastic current is in agreement with previous theoretical [21] as well as experimental findings [20], and shows that the el-ph interactions are essential for model 2.

\section{Bias dependence}

The results in Fig. 2 show that the current in model 1 is mainly governed by the noninteracting current. This implies that the strong QI effects illustrated in Fig. 1(e) are

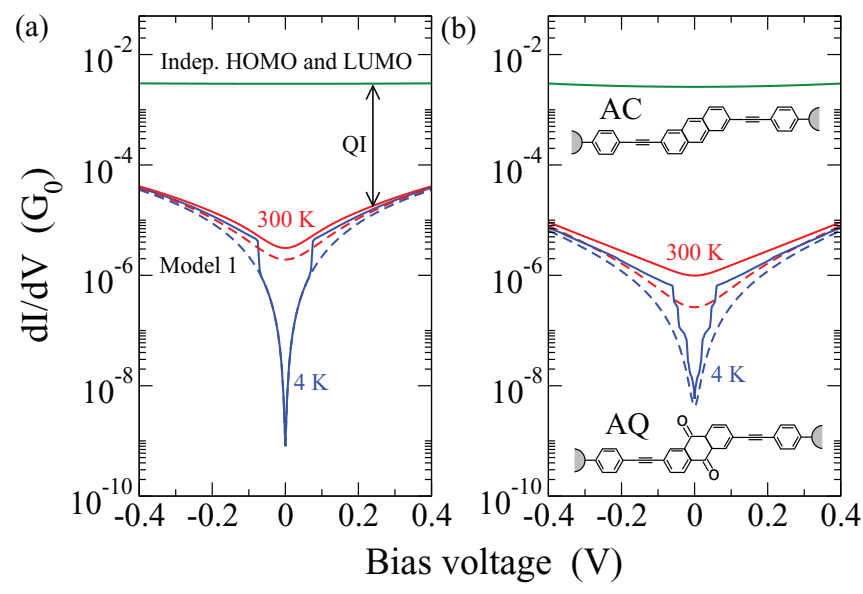

FIG. 3. (Color online) $d I / d V$ vs bias voltage for (a) model 1 and for (b) AQ and AC. Blue curves correspond to $T=4 \mathrm{~K}$ and red curves to $T=300 \mathrm{~K}$. Solid lines include el-ph interactions, while dashed lines are for the noninteracting case. Green curves correspond to (a) the independent HOMO and LUMO model and (b) AC, both including el-ph interactions at $300 \mathrm{~K}$.

robust against finite temperatures and bias voltages. This conclusion is further supported in Fig. 3(a), showing $d I / d V$ versus bias voltage for model 1 . The transmission dip from Fig. 1(e) is clearly recognized at low temperatures and low-bias voltages. When the bias voltage exceeds the phonon energy, a pronounced jump in conductance by almost one order of magnitude is seen at low temperatures. At voltages $V>\hbar \omega / e$ an incoming electron can scatter inelastically by emitting a phonon. This opens up an inelastic transport channel which, at intermediate voltages $0.1<V \lesssim 0.3 \mathrm{~V}$, carries a larger part of current than the noninteracting (elastic) channel. At elevated temperatures, some of the incoming electrons are thermally excited to high enough energies to emit phonons even at zero bias. The abrupt jump in conductance is smeared out, but the characteristic V-shaped $d I / d V$ is still visible. For comparison we show also the conductance of the independent HOMO and LUMO model (solid green). The difference between this and the red/blue curves is due to QI, as also discussed above.

\section{Anthraquinone (AQ) versus anthracene (AC)}

We now consider two similar molecules: the crossconjugated anthraquinone-based molecule (AQ) and the linearly conjugated anthracene-based molecule (AC). The AC and AQ molecules have been studied experimentally in three different works, showing clear signatures of QI in AQ but not in $\mathrm{AC}$ [1-3]. In Fig. 3(b) we show $d I / d V$ versus bias voltage for $\mathrm{AQ}$ and $\mathrm{AC}$ calculated with $\mathrm{DFT}$ within the LOE approximation. For the LOE calculations we include all electronic states and all the phonon modes. Since we have no explicit information about the electrode Fermi energies, we set $E_{F}$ to be exactly at the AQ transmission node [see Fig. 4(a)]. For AC, we set $E_{F}$ between the HOMO and LUMO energies. Experimentally, the alignment between $\mathrm{MO}$ energies and $E_{F}$ can, to some degree, be controlled with a gate electrode, or alternatively with chemical substitutions [29]. 

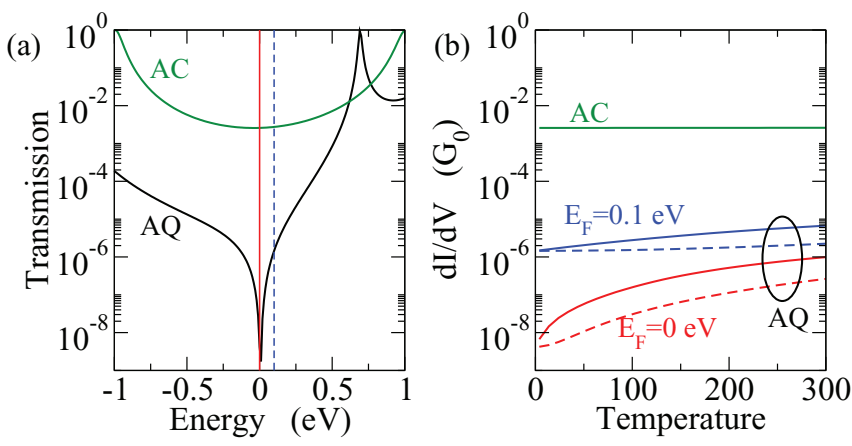

FIG. 4. (Color online) Zero-bias conductance vs temperature for $\mathrm{AQ}$ and $\mathrm{AC}$ with el-ph interactions (solid lines) and without interactions (dashed lines). For AQ we show results for two different values of the Fermi energy. The conductances have been calculated with the LOE approximation to SCBA.

In Fig. 3(b) we essentially see no bias or temperature dependence for $\mathrm{AC}$ (solid green) and the interacting and noninteracting calculations give almost identical results. This situation resembles the independent HOMO- and LUMO model in Fig. 3(a). The trends for AQ are similar to the results from model 1: At low temperatures one observes abrupt increases in conductance at bias voltages corresponding to certain vibrational energies, where inelastic transport channels are opened up. At higher temperatures the sharp features are smeared out, but the el-ph interactions lead to a significantly increased conductance when compared to the noninteracting case (dashed lines). This result is the essence of our findings: Due to the destructive QI effects, one may expect very significant bias- and temperature-dependent conductances; however, the QI effects are not at all completely washed out, and the orders of magnitude difference between linearly and cross-conjugated molecules remains even at finite temperatures.

We finally take a closer look at the temperature dependence of the zero-bias conductance in AQ and AC. Figure 4(b) shows the temperature variation with el-ph interactions (solid lines) and in the noninteracting case (dashed lines). For AQ we show results corresponding to two different positions of the Fermi energy, as indicated by vertical lines in the transmission plot in Fig. 4(a). $E_{F}=0 \mathrm{eV}$ is right at the QI transmission node, and $E_{F}=0.1 \mathrm{eV}$ is slightly above. $\mathrm{AC}$ has a temperature-independent conductance both for the interacting and noninteracting calculations. This is a typical experimental signature of coherent transport. For AQ with $E_{F}=0 \mathrm{eV}$, the noninteracting case yields a $d I / d V \propto T^{2}$ dependence, which can also be derived analytically as follows: Around the QI node, the transmission function can be written as $\mathcal{T}(E)=a E^{2}$. This follows from the Landauer transmission formula, which we may write $\mathcal{T}(E)=\Gamma^{2}\left|G_{1 N}(E)\right|^{2}$, assuming that the molecule is connected to the left and right electrodes at sites 1 and $N[19,30]$. Here $G_{1 N}(E)$ is the $(1, N)$ th element in the retarded Green's function matrix. The transmission node thus comes from a node in $G_{1, N}(E)$. Close to the energy of the node (here assumed to be $E=0$ ) we Taylor expand and write $G_{1, N}(E) \approx \alpha E$, and the quadratic energy dependence of $\mathcal{T}(E)$ follows. The temperature dependence of the conductance can now be obtained as

$$
\begin{aligned}
G(T) & =\frac{2 e^{2}}{h} \int_{-\infty}^{\infty} \mathcal{T}(E)\left(-\frac{\partial f(E, T)}{\partial E}\right) d E \\
& =\frac{2 e^{2} a \beta}{h} \int_{-\infty}^{\infty} E^{2} \frac{e^{\beta E}}{\left(e^{\beta E}+1\right)^{2}} d E \\
& =\frac{2 e^{2} a}{h \beta^{2}} \int_{-\infty}^{\infty} x^{2} \frac{e^{x}}{\left(e^{x}+1\right)^{2}} d x,
\end{aligned}
$$

where $\beta=1 /\left(k_{B} T\right)$. Since the integral is independent of temperature, it follows that $G(T) \propto T^{2}$.

The quadratic temperature dependence, which is only due to the broadening of the Fermi-Dirac distributions, is quite sensitive to the exact location of the transmission node with respect to the Fermi energy, and with $E_{F}=0.1 \mathrm{eV}$, the noninteracting conductance only increases by a factor of 1.5 when increasing the temperature from 4 to $300 \mathrm{~K}$. When el-ph interactions are included, the conductance increases more with temperature than in the noninteracting case for both values of the Fermi level. However, as already mentioned above, the el-ph interactions do not completely destroy the QI effects and at $300 \mathrm{~K}$ there is still a $\sim 3$ orders of magnitude difference in conductance between $\mathrm{AC}$ and $\mathrm{AQ}$.

We note that the increase by several orders of magnitude of the low-bias conductance versus temperature for $A Q$ is similar in magnitude to very recent experimental results for AQ-based devices [6]. We also emphasize that the very strong relative inelastic signals seen for AQ are not a result of particularly strong el-ph interactions for $\mathrm{AQ}$, but are only a result of the very low conductance in the noninteracting case.

\section{CONCLUSION}

In conclusion, we have studied the effect of electron-phonon interactions and finite temperatures on destructive QI effects occurring in molecular junctions. The el-ph interactions have a large relative effect on the conductance in molecules with QI effects due to the low (noninteracting) conductance. This potentially leads to strong temperature dependences on the conductance, as recently observed experimentally [6]. We find, however, that the QI effects are not at all destroyed at room temperature, and when compared to analogous molecules without QI effects, the orders of magnitude difference in conductance remains, in agreement with several recent experiments.

\section{ACKNOWLEDGMENTS}

We thank Mikkel Strange for inspiring discussions. The authors acknowledge support from the Danish Council for Independent Research's Sapere Aude Program through Grants No. 11-104592, No. 11-120938, and No. 11-1051390.

\section{APPENDIX A: VALIDATION OF THE LOE}

Figure 5 shows the current versus voltage for model 1 (top) and model 2 described in the main text. First, the noninteracting current $J_{0}$ (dashed red) is seen to coincide with the elastic current $J_{\mathrm{el}}$ (black triangles), both calculated from Eqs. (4) and (5) in the main text, but using the noninteracting and interacting retarded and/or advanced Green's functions, respectively. The 

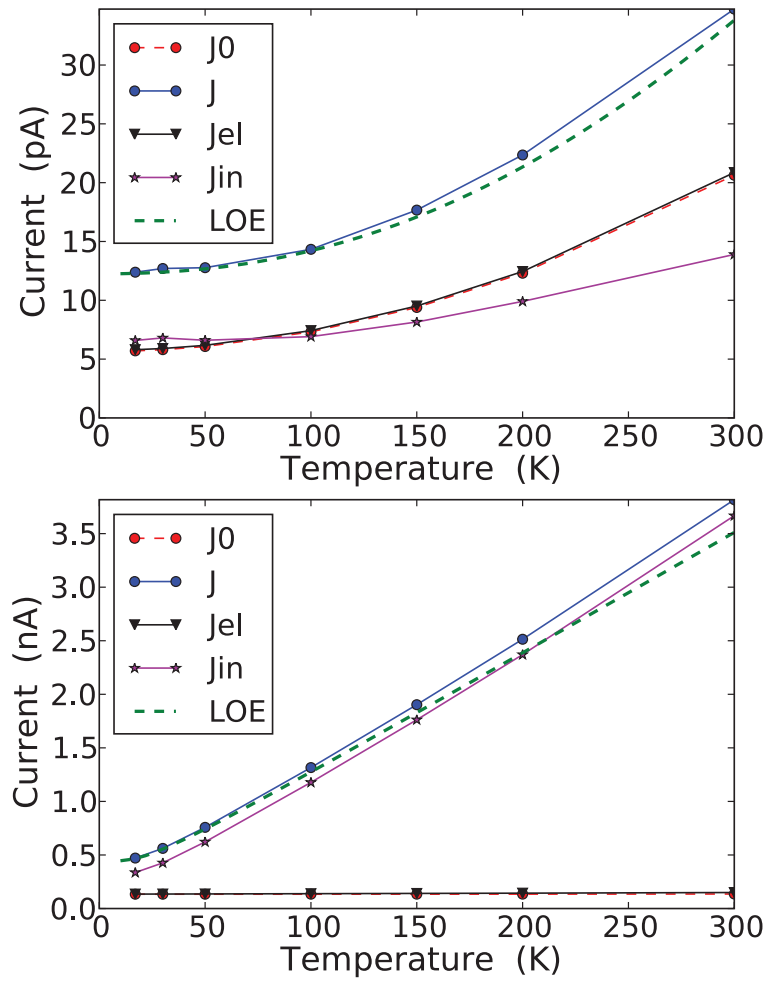

FIG. 5. (Color online) Current vs temperature for model 1 (top) and model 2 (bottom). The bias voltage is $0.1 \mathrm{~V} . J_{0}$ is the noninteracting current, $J$ is the total current calculated with SCBA, which has an elastic $\left(J_{\mathrm{el}}\right)$ and an inelastic $\left(J_{\mathrm{in}}\right)$ contribution. The currents are calculated without the Hartree diagram and are compared with the LOE results (dashed green).

total current $J$ (blue circles) calculated from SCBA is seen to be closely reproduced by the LOE calculation (dashed green). The inelastic contribution to the current, $J_{\text {in }}=J-J_{\text {el }}$, is show as magenta colored stars.

The close agreement between SCBA and LOE allows us to use the computationally much less expensive LOE to study the relatively large $\mathrm{AQ}$ and $\mathrm{AC}$ molecules in the main text.

\section{APPENDIX B: CONSTRUCTION OF SIMPLE MODELS}

In the main text we represent metaconnected benzene and DMBP with two simple two-site models, with model parameters derived from DFT. Here we show how to obtain these parameters.

\section{Benzene: Model 1}

Figure 6 (left column) illustrates the benzene HOMO $\left(\psi_{H}\right)$ and LUMO $\left(\psi_{L}\right)$ Kohn-Sham orbitals as obtained with DFT. The right column shows the linear combinations $\psi_{+}=\left(\psi_{H}+\right.$ $\left.\psi_{L}\right) / \sqrt{2}$ (top) and $\psi_{-}=\left(\psi_{H}-\psi_{L}\right) / \sqrt{2}$ (bottom). In the MO eigenstate basis, the Hamiltonian of the subspace spanned by the $\psi_{H}$ and $\psi_{L}$ is

$$
\mathbf{H}^{(\mathrm{MO})}=\left(\begin{array}{cc}
\varepsilon_{H} & 0 \\
0 & \varepsilon_{L}
\end{array}\right),
$$
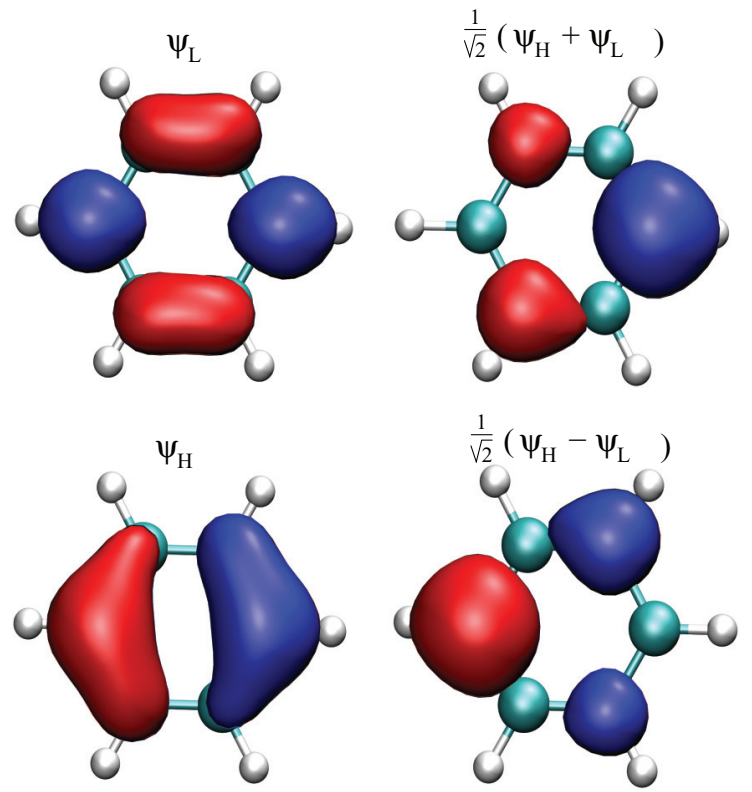

FIG. 6. (Color online) Isosurface plots of the benzene HOMO and LUMO (left column). The right column shows the LMOs formed by addition and subtraction of the HOMO and LUMO. For a metaconnection, one of the LMOs will be coupled to both electrodes, while the other LMO will be decoupled.

where $\varepsilon_{H}$ and $\varepsilon_{L}$ are the HOMO and LUMO orbital energies. In the basis of $\psi_{+}$and $\psi_{-}$(AO basis), the Hamiltonian becomes

$$
\mathbf{H}^{(\mathrm{AO})}=\left(\begin{array}{cc}
\varepsilon_{0} & t \\
t & \varepsilon_{0}
\end{array}\right),
$$

where $\varepsilon_{0}=\left(\varepsilon_{H}+\varepsilon_{L}\right) / 2$ and $t=\left(\varepsilon_{H}-\varepsilon_{L}\right) / 2$.

Figure 6 shows that for a metaconnected benzene, only one of $\psi_{+}$and $\psi_{-}$will have orbital weights on the sites connecting to the leads, while the other will have a large weight. From this we derive model 1 in the main text, where one site (orbital) $(|1\rangle)$ is connected to both leads, while the other is decoupled
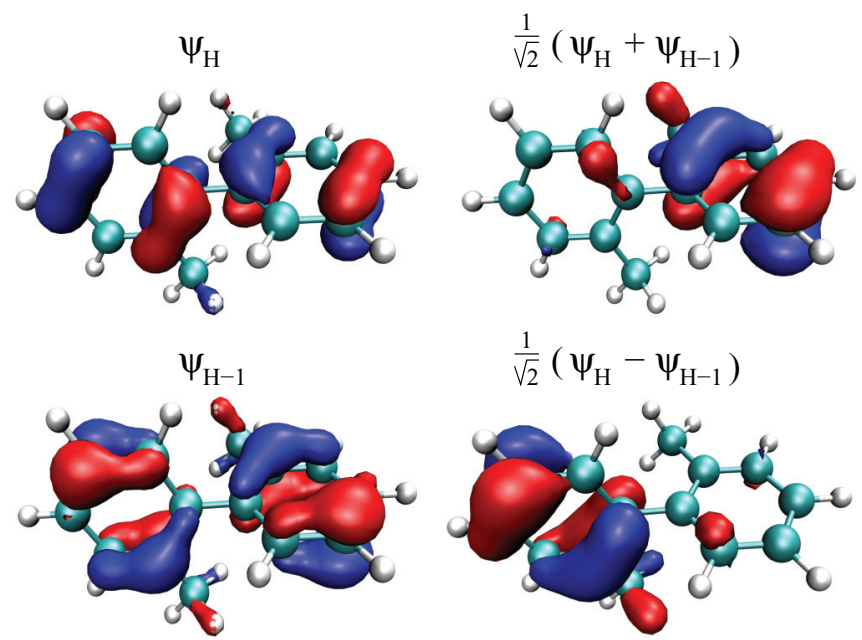

FIG. 7. (Color online) Isosurface plots of the DMBP HOMO and HOMO-1 (left column). The right column shows the localized MOs formed by addition and subtraction of the HOMO and HOMO-1. 
from the leads. Model 1 closely resembles the situation of a Fano resonance [31], where a localized state couples to a continuum. It can easily be shown that the (noninteracting) transmission function always will have a node at the energy of the localized site [15].

\section{DMBP: Model 2}

Figure 7 illustrates the HOMO and HOMO-1 (left column) of DMBP as obtained with DFT. The right column shows the linear combinations $\psi_{+}=\left(\psi_{H}+\psi_{H-1}\right) / \sqrt{2}$ (top) and $\psi_{-}=$
$\left(\psi_{H}-\psi_{H-1}\right) / \sqrt{2}$ (bottom). In the basis of $\psi_{+}$and $\psi_{-}$(AO basis) we obtain a Hamiltonian similar to Eq. (B2) with $\varepsilon_{0}=$ $\left(\varepsilon_{H}+\varepsilon_{H-1}\right) / 2$ and $t=\left(\varepsilon_{H}-\varepsilon_{H-1}\right) / 2$. Since the HOMO and HOMO-1 states are nearly degenerate, the coupling matrix element $t$ is small.

From the left and right localized $\psi_{+}$and $\psi_{-}$we derive model 2: $\psi_{-}$[site $|1\rangle$ in Fig. 1(d)] is only coupled to the left electrode since the orbital weight is much larger on the left part of the molecule than on the right, while $\psi_{-}$is only connected to the right electrode.
[1] D. Fracasso, H. Valkenier, J. C. Hummelen, G. C. Solomon, and R. C. Chiechi, J. Am. Chem. Soc. 133, 9556 (2011).

[2] W. Hong, H. Valkenier, G. Meszaros, D. Z. Manrique, A. Mishchenko, A. Putz, P. M. Garcia, C. J. Lambert, J. C. Hummelen, and T. Wandlowski, Beilstein J. Nanotechnol. 2, 699 (2011).

[3] C. M. Guedon, H. Valkenier, T. Markussen, K. S. Thygesen, J. C. Hummelen, and S. J. van der Molen, Nat. Nanotechnol. 7, 305 (2012).

[4] S. V. Aradhya, J. S. Meisner, M. Krikorian, S. Ahn, R. Parameswaran, M. L. Steigerwald, C. Nuckolls, and L. Venkataraman, Nano Lett. 12, 1643 (2012).

[5] C. R. Arroyo, S. Tarkuc, R. Frisenda, J. S. Seldenthuis, C. H. M. Woerde, R. Eelkema, F. C. Grozema, and H. S. J. van der Zant, Angew. Chem. 125, 3234 (2013).

[6] V. Rabache, J. Chaste, P. Petit, M. L. Della Rocca, P. Martin, J.-C. Lacroix, R. L. McCreery, and P. Lafarge, J. Am. Chem. Soc. 135, 10218 (2013).

[7] C. Patoux, C. Coudret, J. P. Launay, C. Joachim, and A. Gourdon, Inorg. Chem. 36, 5037 (1997).

[8] M. Mayor, H. B. Weber, J. Reichert, M. Elbing, C. von Hänisch, D. Beckmann, and M. Fischer, Angew. Chem. Int. Ed. 42, 5834 (2003).

[9] H. Valkenier, C. M. Guédon, T. Markussen, K. S. Thygesen, S. J. van der Molen, and J. C. Hummelen, Phys. Chem. Chem. Phys. 16, 653 (2014).

[10] T. Markussen, J. Schiötz, and K. S. Thygesen, J. Chem. Phys. 132, 224104 (2010).

[11] N. Darwish, I. Diez-Perez, P. Da Silva, N. Tao, J. J. Gooding, and M. N. Paddon-Row, Angew. Chem. 124, 3257 (2012).

[12] J. P. Bergfield and C. A. Stafford, Nano Lett. 9, 3072 (2009).

[13] C. M. Finch, V. M. Garcia-Suarez, and C. J. Lambert, Phys. Rev. B 79, 033405 (2009).

[14] J. P. Bergfield, M. A. Solis, and C. A. Stafford, ACS Nano 4, 5314 (2010).
[15] R. Stadler and T. Markussen, J. Chem. Phys. 135, 154109 (2011).

[16] N. F. Phelan and M. Orchin, J. Chem. Educ. 45, 633 (1968).

[17] G. C. Solomon, C. Herrmann, T. Hansen, V. Mujica, and M. A. Ratner, Nat. Chem. 2, 223 (2010).

[18] G. C. Solomon, D. Q. Andrews, R. H. Goldsmith, T. Hansen, M. R. Wasielewski, R. P. Van Duyne, and M. A. Ratner, J. Am. Chem. Soc. 130, 17301 (2008).

[19] T. Markussen, R. Stadler, and K. S. Thygesen, Nano Lett. 10, 4260 (2010).

[20] S. Ballmann, R. Hartle, P. B. Coto, M. Elbing, M. Mayor, M. R. Bryce, M. Thoss, and H. B. Weber, Phys. Rev. Lett. 109, 056801 (2012).

[21] R. Hartle, M. Butzin, O. Rubio-Pons, and M. Thoss, Phys. Rev. Lett. 107, 046802 (2011).

[22] R. Hartle, M. Butzin, and M. Thoss, Phys. Rev. B 87, 085422 (2013).

[23] M. Galperin, M. A. Ratner, and A. Nitzan, J. Phys.: Condens. Matter 19, 103201 (2007).

[24] T. Frederiksen, M. Paulsson, M. Brandbyge, and A.-P. Jauho, Phys. Rev. B 75, 205413 (2007).

[25] J. Enkovaara, C. Rostgaard, J. J. Mortensen, J. Chen, M. Dulak, L. Ferrighi, J. Gavnholt, C. Glinsvad, V. Haikola, H. A. Hansen et al., J. Phys.: Condens. Matter 22, 253202 (2010).

[26] K. Kaasbjerg, K. S. Thygesen, and K. W. Jacobsen, Phys. Rev. B 85, 115317 (2012).

[27] Y. Meir and N. S. Wingreen, Phys. Rev. Lett. 68, 2512 (1992).

[28] M. Paulsson, T. Frederiksen, and M. Brandbyge, Phys. Rev. B 72, 201101 (2005).

[29] L. Venkataraman, Y. S. Park, A. C. Whalley, C. Nuckolls, M. S. Hybertsen, and M. L. Steigerwald, Nano Lett. 7, 502 (2007).

[30] T. Markussen, R. Stadler, and K. S. Thygesen, Phys. Chem. Chem. Phys. 13, 14311 (2011).

[31] A. E. Miroshnichenko, S. Flach, and Y. S. Kivshar, Rev. Mod. Phys. 82, 2257 (2010) 